\title{
Follow-up of children with antenatally diagnosed idiopathic polyhydramnios
}

\author{
Magdalena Adamczyk, Jakub Kornacki, Przemyslaw Wirstlein, \\ Malgorzata Szczepanska, Ewa Wender-Ozegowska
}

Division of Reproduction, Department of Gynecology and Obstetrics, Poznan University of Medical Sciences, Poland

\begin{abstract}
Objectives: The aim of our work was to assess the development of children with antenatally diagnosed idiopathic polyhydramnios, over 12 months from the end of pregnancy.

Material and methods: The study included 91 healthy pregnant patients with idiopathic polyhydramnios. Diagnostic tests results and perinatal medical history were obtained retrospectively. Parents of children were contacted by phone and by mail. The answers were obtained from 64 (70\%) parents. For statistical analysis SigmaStat3.5 software was used.

Results: Ninety six percent of parents declared that in their opinion the development of children was normal. Abnormalities were found in $44 \%$ of the children. Thirty percent of neonates demonstrated mild abnormalities which may be due to organic or functional neuromuscular disorders: abnormal muscle tone, speech apparatus and development disorders, swallowing and breathing problems (manifested as vomiting, excessive regurgitation, idiopathic apnoeas).

Isolated small malformations were diagnosed in 12 (19\%) children. Two children (3\%) with SGA were diagnosed with genetic syndromes. More than one of the abnormalities described above were diagnosed in $14 \%$ of children. Gestational age at the time of polyhydramnios diagnosis and its severity were not prognostic factors for abnormalities. Seventy percent of newborns were male.

Conclusions: Despite the subjectively positive assessment of the development of children by the majority of parents, groups of common disorders requiring long-term follow-up have been identified. Functional disorders of the gastrointestinal tract, CNS and the group of neuromuscular disorders may be responsible for idiopathic polyhydramnios. SGA with co-existing idiopathic polyhydramnios is associated with the risk of genetic diseases. The more frequent incidence of idiopathic polyhydramnios in male fetuses requires further research.

Key words: congenital anomalies; development; functional disorders; idiopathic polyhydramnios; neuromuscular disorders
\end{abstract}

Ginekologia Polska 2019; 90, 2: 93-99

\section{INTRODUCTION}

Polyhydramnios is involved in 1-2\% of pregnancies [1]. Congenital defects, aneuploidy, maternal diabetes, isoimmunization diseases, intrauterine infections, multiple pregnancies and placental tumours are causes of polyhydramnios. Prenatal diagnostics for fetal defects, chromosomal aberrations, gestational diabetes, immunization, selected viral and parasitic infections is a necessary procedure in the case of abnormal, increased amount of amniotic fluid. About $50-60 \%$ of cases of polyhydramnios have the unknown etiology [2]. This condition is called idiopathic polyhydramnios.

Polyhydramnios is associated with the risk of complications and adverse perinatological outcomes, such as: premature births, premature rupture of the membranes, premature abruptio of the placenta, prolapse of the umbilical cord, operative delivery by caesarean section or postpartum atony of the uterus.

In most cases of idiopathic polyhydramnios, the mechanism responsible for the excessive accumulation of amniotic fluid remains unclear. The underlying cause, which is organic, functional or a consequence of the impaired distribution of the amniotic fluid in the amniotic cavity, may be difficult to diagnose using available diagnostic methods. The increased production or reduced absorption of amniotic fluid, as well as a combination of both these causes [3-6] may be responsible for the disturbance of the balance of the amniotic fluid circulation.

We currently have little knowledge about the development of children with the history of idiopathic polyhydramnios during pregnancy. In these cases, prenatal counselling 
is difficult, and prognosis about the health of the child is unpredictable. The lack of reliable data in this regard is due to, among others, the necessity to refer to the results of research from the last dozen or even several dozen years in which methods and diagnostic procedures were less sensitive than those used today. The rapid advancement of technology and the consequent higher quality of ultrasound devices gives us a much greater possibility of detailed imaging diagnostics, while improved neonatological procedures allow for a high standard of postnatal care.

The aim of our work is to assess the development of children within 12 months to 8 years from the end of pregnancy complicated by idiopathic polyhydramnios.

The knowledge about the fate of these children may help to identify new causes of polyhydramnios and give an opportunity to develop new prenatal diagnostic methods and appropriate obstetric and perinatal management.

\section{MATERIAL AND METHODS}

The study design was approved by the Ethics Committee of the Karol Marcinkowski University of Medical Sciences in Poznan, Poland (\#701/18 on 14-06-2018).

The study included 185 patients with polyhydramnios hospitalized in the second and third trimester of pregnancy at the Gynaecology and Obstetrics Department of the Clinical Hospital in Poznan in the years 2008-2015.

The AFI (Amniotic Fluid Index) $>20 \mathrm{~cm}$ was accepted as the criterion for diagnosis of polyhydramnios [7]. Mild polyhydramnios was diagnosed with $\mathrm{AFI}<30 \mathrm{~cm}$ and severe with $\mathrm{AFI} \geq 30 \mathrm{~cm}$.

During the hospitalization at the Department, the patients were subjected to diagnostic procedures to identify possible causes of polyhydramnios. Ultrasound examination with the biometric evaluation and the detailed assessment of fetal anatomy and the severity of polyhydramnios was performed in all patients. Imaging examinations were carried out by the same, qualified team of ultrasound specialists with many years of experience in fetal diagnostics. In justified cases, if fetal anaemia was suspected, the diagnosis was extended to include TORCH tests, serological examinations to identify alloantibodies against red blood cell antigens and the assessment of middle cerebral artery peak systolic velocity (MCA PSV). Screening and diagnostic tests for gestational diabetes were reinterpreted. All patients with a prenatally diagnosed probable cause of polyhydramnios were excluded from further analysis.

Demographic data, information about the obstetric past, current pregnancy history, diagnostic tests results and perinatal medical history were obtained retrospectively on the basis of hospitalization medical records.

Idiopathic polyhydramnios was diagnosed in 91 healthy pregnant patients enrolled in the study.
Parents of children who were qualified to participate in the study were contacted by phone and by mail to get information on the development and health status of children over 1 year of age. The medical history included defects diagnosed after birth, malformations, causes of possible prolonged hospitalization, development of children in infancy and early childhood, including motor and intellectual skills, the necessity of rehabilitation and other specialist medical care, results of diagnostic examinations, operations and hospitalizations.

The answers were obtained from 64 (70\%) parents qualified to participate in the study. Eighty percent of them provided the answer by phone, the remaining 13 parents sent a letter response.

The endpoints of the study were the mortality rate, percentage of postnatally diagnosed congenital malformations, genetic syndromes, metabolic and neurological diseases, including cognitive and motor disorders.

\section{Statistical analysis}

In order to assess the significance of differences observed between variables in the study groups, Student's $t$-test was used and univariate analysis of variance (ANOVA) with the Holm-Sidak multiple repeat test for variables with normal distribution and their rank versions for variables with non-parametric distribution. In order to evaluate the differences in the distribution of non-quantitative variables, the Fisher-Freeman-Halton exact test, Fisher's exact test for $2 \times 2$ tables and Chi-square test were applied. The $p$ value $<0.05$ was accepted as statistically significant.

\section{RESULTS}

The analysis covered the development and fate of 64 children with the obstetric history of idiopathic polyhydramnios. Seventy five percent of the pregnancies were found with mild polyhydramnios $(\mathrm{AFI}<30 \mathrm{~cm})$, in 16 patients AFI exceeded $30 \mathrm{~cm}$ and polyhydramnios was classified as severe.

Idiopathic polyhydramnios was most often diagnosed in the second half of the third trimester (approximately in the $33^{\text {rd }}$ week of gestation on average). Nearly $90 \%$ of pregnancies ended with birth at term. Seven births took place before the completion of the 37th week of gestation. The percentage of caesarean sections was $51 \%$. The birth weight of $84 \%$ of newborns was normal, two children (3\%) were diagnosed with small for gestational age (SGA) and macrosomia ( $>4000 \mathrm{~g}$ ) was found in eight newborns. Seventy percent of newborns were male. Perinatological results showing the severity of idiopathic polyhydramnios are presented in Table 1.

The mortality rate was $0 \%$. No abnormalities were found in $56 \%$ of the children. 
Table 1. Demographic and obstetric characteristics of patients with isolated polyhydramnios in relation to polyhydramnios severity

\begin{tabular}{|c|c|c|c|}
\hline & $\begin{array}{l}\mathrm{AFI}<30 \\
n=48\end{array}$ & $\begin{array}{l}A F I \geq 30 \\
n=16\end{array}$ & p-value \\
\hline $\begin{array}{l}\text { Maternal age (years) } \\
\text { Median (range) }\end{array}$ & $31(17-40)$ & $31(23-37)$ & 0.608 \\
\hline $\begin{array}{l}\text { Gestational age at } \\
\text { diagnosis (week) } \\
\text { Median (range) }\end{array}$ & $34(25-39)$ & $33(24-40)$ & 0.870 \\
\hline Early 20-29 wk & 11 & 4 & \multirow{3}{*}{0.814} \\
\hline Medium 30-34 wk & 15 & 6 & \\
\hline Late $+35 w k$ & 22 & 6 & \\
\hline $\begin{array}{l}\text { Gestational age } \\
\text { at delivery (week) } \\
\text { Median (range) }\end{array}$ & $39(32-42)$ & $40(35-42)$ & 0.224 \\
\hline \multicolumn{4}{|l|}{ Delivery mode (\%) } \\
\hline $\begin{array}{l}\text { Spontaneous vaginal } \\
\text { delivery }\end{array}$ & $22(45.8)$ & $4(25.0)$ & \multirow{3}{*}{0.299} \\
\hline Cesarean section (CC) & $22(45.8)$ & $11(68.8)$ & \\
\hline Vacuum extractor (VE) & $4(8.4)$ & $1(6.2)$ & \\
\hline $\begin{array}{l}\text { Newborns birth weight }[g] \\
\text { Mean }( \pm \text { SD) }\end{array}$ & $3546( \pm 590)$ & $3502( \pm 535)$ & 0.793 \\
\hline $\begin{array}{l}\text { Macrosomy (> } 4000 \mathrm{~g}) \\
\mathrm{n}(\%)\end{array}$ & $6(12.5)$ & $2(12.5)$ & 1.000 \\
\hline \multicolumn{4}{|l|}{ Sex, $n(\%)$} \\
\hline Male & $35(72.9)$ & $10(62.5)$ & \multirow{2}{*}{0.530} \\
\hline Female & $13(27.1)$ & $6(37.5)$ & \\
\hline
\end{tabular}

Two children were diagnosed with genetic syndromes - Down syndrome and Rubinstein-Taybi syndrome (RTS), however the diagnosis of RTS was based on the clinical presentation. The low birth weight of children with genetic syndromes was a premise to make the diagnosis of SGA.

Isolated small malformations were diagnosed in 12 children, which constituted over $19 \%$ of newborns without genetic syndromes. Congenital anomalies included various systems. Among malformations important in terms of polyhydramnios we can indicate: an unspecified anomaly of the central nervous system, mandibular defect and hypospadias.

Thirty percent of neonates born in a good condition, with the normal Apgar score demonstrated abnormalities which may be due to organic or functional neuromuscular disorders: abnormal muscle tone, speech apparatus and development disorders, swallowing and breathing problems (manifested as vomiting, excessive regurgitation, idiopathic apnoeas). The most numerous group were children requiring rehabilitation due to the abnormal muscle tone (14.5\%).

Approximately $5 \%$ of children were deficient in growth and body weight adequate to a given calendar age, despite the normal birth weight; two (3\%) children required intensive treatment due to haemolytic disease caused by the incompatibility in the AB0 blood group system of the isoimmunization etiology.

More than one of the abnormalities described above were diagnosed in $14 \%$ of children. Abnormalities diagnosed in children after childbirth and their frequency, broken down into proposed groups of disorders, are presented in Table 2.

In the group of healthy children and those with abnormalities, including multiple defects, gestational age at the time of polyhydramnios diagnosis and its severity were not prognostic factors. SGA was associated with the risk of numerous genetic abnormalities. The percentage of healthy children with macrosomia was similar to that of children with neuromuscular disorders. The boys made up 56\% of healthy children. A disproportion in the sex distribution was clear in the group of children with neuromuscular disorders and multiple abnormalities ( 82 vs. $18 \%$ and 78 vs. $22 \%$ ). The abnormal muscle tension and the need for rehabilitation were reported only in boys. Perinatological results in the group of healthy children and those with diagnosed abnormalities are presented in Table 3.

\section{DISCUSSION}

The causes of polyhydramnios could not be determined in $49 \%$ of patients hospitalized in our Department. This percentage is lower than described in the literature (50-70\%). No significant congenital defect, which could be diagnosed prenatally, was overlooked in diagnostic imaging. An experienced team of ultrasound specialists and perinatal medicine specialists provides the high quality of prenatal diagnosis, burdened with a low risk of diagnostic failures.

Research on the fate of children with the medical history of idiopathic polyhydramnios, discussed below, used mainly medical databases, including medical opinions and diagnoses based on diagnostic procedures. The medical history taken from children's carers is burdened with the risk of misinterpreting medical information received from the primary care physician and specialists, and often proves the lack of any diagnostics. Undoubtedly, however, the subjective assessment of the child's development made by parents and their expectations are an advantage of the study. On the basis of phone conversations and letter correspondence, $96 \%$ of parents declared that in their opinion the development of children was normal. However, a detailed, rigorous analysis of the information allowed to formulate conclusions and hypotheses on the possible causes and consequences of the increased amount of amniotic fluid in pregnancy.

At the beginning, we would like to answer the question: does the normal weight of the child or its disorders, such as SGA or macrosomia accompanying idiopathic polyhydramnios may be a prognostic factor of abnormalities after delivery? 
Table 2. Disorders diagnosed after birth in children with idiopathic polyhydramnios

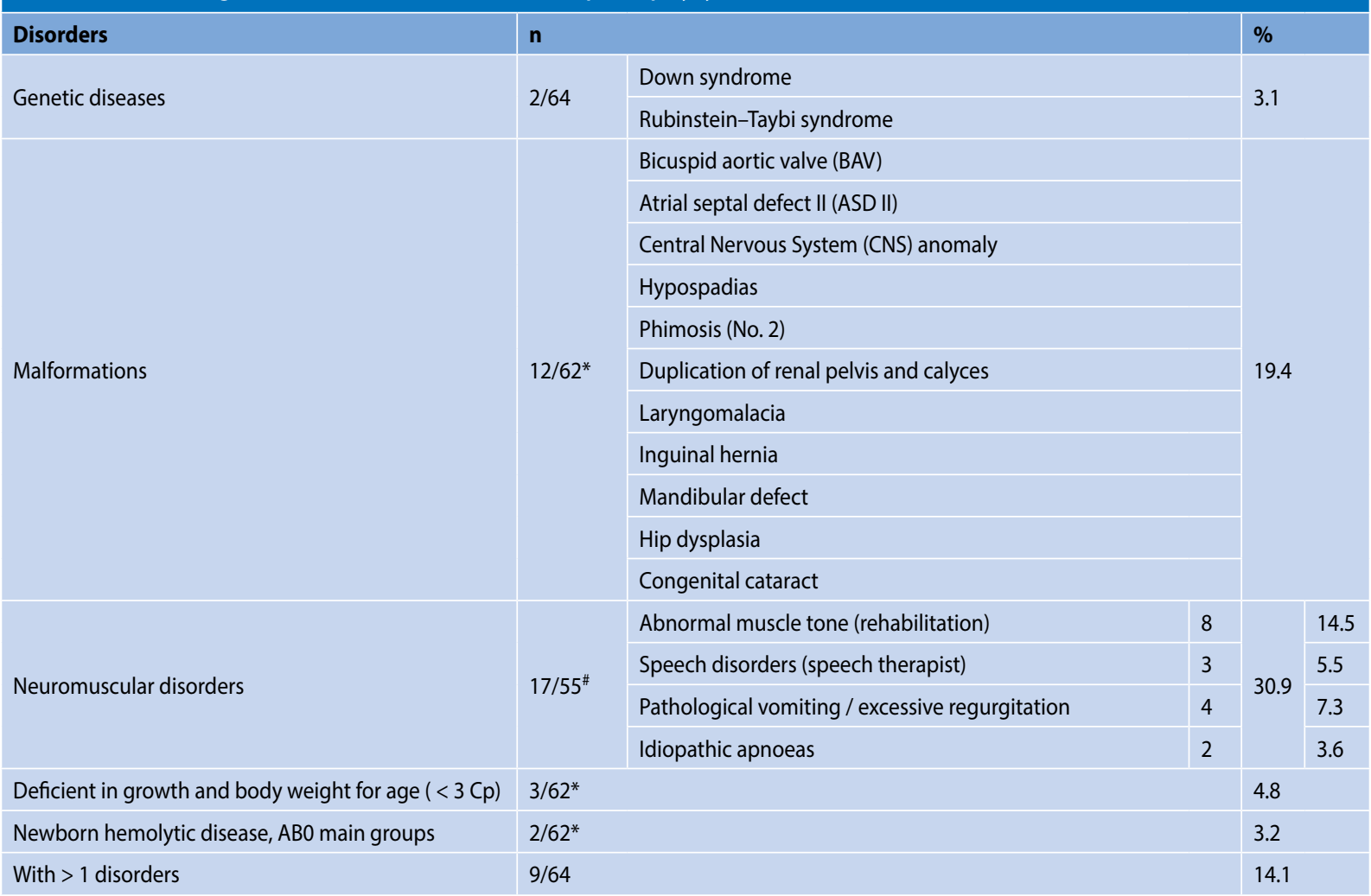

*Number of children, excluding children with genetic diseases; ${ }^{*}$ Number of children, excluding children with genetic diseases and those born prematurely

The percentage of fetuses with macrosomia in our study was $12.5 \%(8 / 64)$ and was similar to the results obtained by Yefet [8]. Yefet showed a statistically significant relationship between fetal macrosomia and idiopathic polyhydramnios, and compared the outcome to the control group without polyhydramnios ( $11 \%$ vs. $5 \%$ ). The study conducted by Dorleijn et al. found fetal macrosomia (> $4000 \mathrm{~g}$ ) coexisting with idiopathic polyhydramnios in $25 \%$ of cases and was associated with a good prognosis [9]. Unlike the Dorleijn's results, our analysis revealed that the risk of abnormalities after delivery in children with macrosomia was the same as in children with the normal body weight.

In all cases of SGA, that we have identified, the prognosis was poor and genetic syndromes were diagnosed in children after birth. Like in our study, Kollmann showed that too low foetus weight and polyhydramnios are risk factors for abnormalities [10].

Abnormalities in children with idiopathic polyhydramnios are a group of heterogeneous disorders. These include: genetic syndromes, developmental malformations (facial defects: cleft lip, cleft palate, Pierre-Robin syndrome), metabolic syndromes (Bartter syndrome - tubulopathy disease), neuromuscular disorders (including those genetically determined as for example myotubular myopathy linked to chromosome $\mathrm{X}$ ) and others [9].
The percentage of genetic defects estimated in our study was $3.1 \%$. This result is similar to the outcomes obtained by Yefet et al. (3.7\%) and the meta-analysis published in 2015 [8, 11]. On the basis of the analysis of 1729 pregnancies with polyhydramnios of the unknown etiology, Sagi-Dain estimated the risk of chromosomal aberration at $2.8 \pm 3.7 \%$. However, he criticized the result obtained, because he believes that the real risk of chromosomal aberrations is lower than estimated. The drawback of this meta-analysis is the lack of assessment of advanced molecular techniques, including microarray methods used in the diagnosis of sub-microscopic chromosome rearrangements as a possible cause of idiopathic polyhydramnios. The genetic syndromes diagnosed after childbirth include not only those conditioned by an abnormal karyotype, but also chromosomal microrearrangements and single gene mutations (Noonan syndrome, Beckwith-Wiedermann syndrome, Shprintzen-Goldberg syndrome) [9].

The average rate of congenital defects in the general population is approximately $5 \%$ [12]. The results of our study showed higher than in the population (19.4\%) percentage of defects in children with the history of idiopathic polyhydramnios. Similarly, the authors of a large retrospective study found twice the risk of congenital malformations in this group of children compared to the control group [8]. 


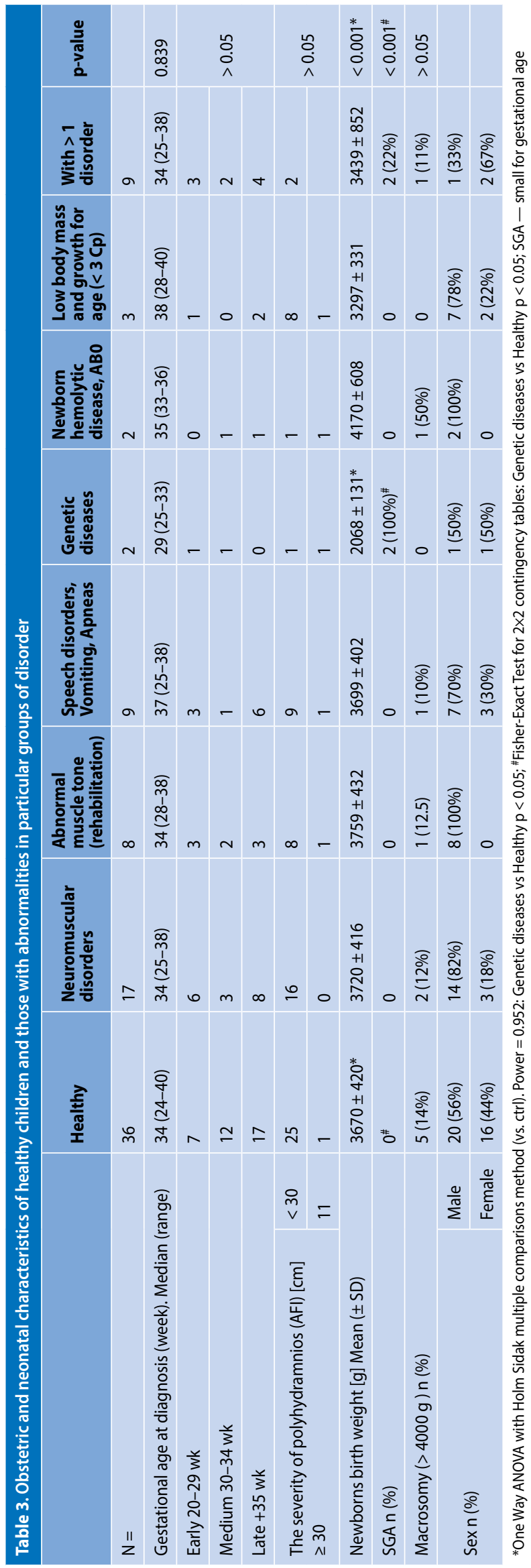

They have noticed, however, that the diagnosis of over $60 \%$ of defects, which were postnatally diagnosed, was still possible during pregnancy. The analysis of defects, including the division into systems, did not allow the authors to indicate a system which is significantly more often affected than others.

The relationship between idiopathic polyhydramnios and neuromuscular disorders is interesting. Our work indicated that $14.5 \%$ of children born at term $(8 / 55)$ with the normal Apgar score needed rehabilitation after delivery due to the abnormal muscle tone. Yefet also showed more than three times higher risk of neurological problems (especially motor disorders) and delayed development in children with the history of idiopathic polyhydramnios compared to the control group (9.7\% vs. $3 \%$ ) [8].

In 2010, Sekulić set an interesting hypothesis on the possible effect of polyhydramnios on the fetal development and ossification [13]. In this concept, the author refers to the results of studies carried out in professional divers. This hypothesis assumes that, by reducing the apparent fetal body weight (from the normal $60-80 \%$ to $10-20 \%$ in polyhydramnios) and mechanical stress, the increased amount of amniotic fluid can lead to disturbances of ossification and the abnormal bone development in the prenatal period. According to the authors, these changes can lead to disorders in the skeletal-muscular system in children, muscle tone problems with the delayed motor development as the clinical manifestation.

We can treat neuromuscular disorders as a cause of polyhydramnios in the mechanism of the abnormal amniotic fluid swallowing reflex. In generalized neuromuscular disorders, we observe the consequences of the increased amount of amniotic fluid, as in the hypothesis described above. Therefore, it should be explained whether neuromuscular disorders are the cause or effect of polyhydramnios?

The distribution of newborn sex is particularly noteworthy. A predominance of male is clearly visible in the group of examined children (70\%). Our last work on non-idiopathic polyhydramnios revealed that the distribution of sex was similar to the one in population (54\% vs. $46 \%$ ), with a slight male predominance [14]. A similar observation was made by Stanescu et al. [15]. In their work, almost $74 \%$ of newborns with idiopathic polyhydramnios were boys. In the commentary to the above publication, Kim indicated the possible association between idiopathic polyhydramnios and nocturnal enuresis [16]. In both cases, these problems more often affect boys. This raises the question of whether it is possible to assess the daily rhythm of urinary output and whether the evaluation of bladder capacity after delivery will help to identify children at risk for nocturnal enuresis in the future?

Noteworthy in our work is that muscular tension disorders, that required rehabilitation after delivery, were found 
only in boys. Sex-linked diseases are determined by the presence of alleles located on the $X$ chromosome. Recessively inherited diseases are manifested in men because they have only one $X$ chromosome. 1184 genes have been identified on the $\mathrm{X}$ chromosome.

Many genetic neurodegenerative and neuromuscular diseases, including those sex-linked, are triggered by a dynamic mutation related to the expansion of trinucleotide repeats [17]. Myotonic dystrophy is also a neurodegenerative disease. The relationship between myotonic dystrophy and idiopathic polyhydramniosis was well documented [18]. According to Rudnik-Schöneborn et al., myotonic dystrophy is the cause of $9.7 \%$ of cases of idiopathic polyhydramnios [19]. Yee $C$ showed that $66 \%$ of children with myotonic dystrophy were diagnosed with idiopathic polyhydramnios and proposed the appropriate diagnostic procedures to be introduced in a situation of polyhydramnios coexisting with a positive family history of dystrophy, improper limb position and the reduction of fetal movements visible in the ultrasound image [20]. Children with the medical history of idiopathic polyhydramnios and deficiency in growth and body weight, suffering from idiopathic apnoeas in the long-term follow-up should undergo detailed diagnostic procedures. We failed to determine causes of these symptoms in all analysed cases.

The etiology of apnoea in premature babies is well known, but in full-term children it remains a challenge. Apnoea may be central, associated with depression of the respiratory centre or obstructive. The coexistence of apnoea and gastroesophageal reflux was reported on numerous occasions, however, the last literature review did not ultimately dispel doubts on the presence of this correlation [21]. Apnoea in infants is still one of possible causes of Sudden Infant Death Syndrome (SIDS) which occurs in the mechanism of aspiration of chyme into the lungs. In our study we did not encounter a similar situation, while in the literature the cases of SIDS were described in children with the medical history of idiopathic polyhydramnios [9].

We did not diagnose a congenital infection (from the TORCH group) in any child. Authors of many separate studies on the relationship between intrauterine infections $(\mathrm{TORCH}$, including parvovirosis) and polyhydramnios agree that such correlation is strongly doubtful. Therefore, it seems that in the cases of unexplained polyhydramnios, the diagnosis for TORCH is unjustified [22].

Summing up, polyhydramnios is a symptom of many pathologies. In the absence of other obstetrical disorder or abnormality in the ultrasound image, functional pathologies should be suspected, resulting in the ineffective amniotic fluid swallowing reflex or excessive urine production. A large group of neuromuscular disorders, central nervous system problem or genetic syndromes without anatomical defects are possible causes of idiopathic polyhydramnios. Diagnostic procedures based on CNS functional imaging (functional magnetic resonance imaging $\mathrm{fMRI}$ ), tests for myopathy (electromyography EMG) and diseases of the peripheral nervous system would allow for an objective assessment of this hypothesis [23].

It is worth noting that, except for children with genetic syndromes, the prognosis in children with idiopathic polyhydramnios in a short several-year observation is good and the accompanying diseases are mild. However, long-term observation is necessary to make a complete assessment.

\section{CONCLUSIONS}

1. Despite the subjectively positive assessment of the development of children by the majority of parents, groups of common disorders requiring long-term follow-up have been identified.

2. Functional disorders of the gastrointestinal tract, central nervous system and the group of neuromuscular disorders may be responsible for idiopathic polyhydramnios.

3. Postnatal diagnosis of neuromuscular disorders and long-term observation is necessary for the objective evaluation of their possible connection with idiopathic polyhydramniosis.

4. SGA with co-existing idiopathic polyhydramnios is associated with the risk of genetic diseases.

5. The more frequent incidence of idiopathic polyhydramnios in male fetuses requires further research.

\section{Conflicts of interest}

The authors have stated explicitly there are no conflicts of interest in connection with this article.

\section{REFERENCES}

1. Volante $E$, Gramellini D, Moretti S, et al. Alteration of the amniotic fluid and neonatal outcome. Acta Biomed. 2004; 75 Suppl 1: 71-75, indexed in Pubmed: 15301296.

2. Magann EF, Chauhan SP, Doherty DA, et al. A review of idiopathic hydramnios and pregnancy outcomes. Obstet Gynecol Surv. 2007; 62(12): 795-802, doi: 10.1097/01.ogx.0000290349.58707.e0, indexed in Pubmed: 18005456.

3. Lee SM, Jun JK, Lee EJ, et al. Measurement of fetal urine production to differentiate causes of increased amniotic fluid volume. Ultrasound Obstet Gynecol. 2010; 36(2): 191-195, doi: 10.1002/uog.7519, indexed in Pubmed: 20069667.

4. Touboul C, Picone O, Levaillant JM, et al. Clinical application of fetal urine production rate in unexplained polyhydramnios. Ultrasound Obstet Gynecol. 2009; 34(5): 521-525, doi: 10.1002/uog.6440, indexed in Pubmed: 19830790.

5. Brace RA, Anderson DF, Cheung CY. Fetal swallowing as a protective mechanism against oligohydramnios and polyhydramnios in late gestation sheep. Reprod Sci. 2013;20(3):326-330, doi:10.1177/1933719112453510, indexed in Pubmed: 22872543.

6. Zhu X, Jiang S, Hu Y, et al. The expression of aquaporin 8 and aquaporin 9 in fetal membranes and placenta in term pregnancies complicated by idiopathic polyhydramnios. Early Hum Dev. 2010; 86(10): 657-663, doi: 10.1016/j.earlhumdev.2010.07.012, indexed in Pubmed: 20732771.

7. Harlev A, Sheiner E, Friger M, et al. Polyhydramnios and adverse perinatal outcome - what is the actual cutoff? J Matern Fetal Neonatal Med. 2014; 27(12): 1199-1203, doi: 10.3109/14767058.2013.853736, indexed in Pubmed: 24111654. 
8. Yefet E, Daniel-Spiegel E. Outcomes From Polyhydramnios With Normal Ultrasound. Pediatrics. 2016; 137(2): e20151948, doi: 10.1542/peds.20151948, indexed in Pubmed: 26755694.

9. Dorleijn DMJ, Cohen-Overbeek TE, Groenendaal F, et al. Idiopathic polyhydramnios and postnatal findings. J Matern Fetal Neonatal Med. 2009; 22(4): 315-320, doi: 10.1080/14767050802531870, indexed in Pubmed: 19085623.

10. Kollmann M, Voetsch J, Koidl C, et al. Etiology and perinatal outcome of polyhydramnios. Ultraschall Med. 2014; 35(4):350-356, doi: 10.1055/s0034-1366115, indexed in Pubmed: 24729436.

11. Sagi-Dain L, Sagi S. Chromosomal aberrations in idiopathic polyhydramnios: A systematic review and meta-analysis. Eur J Med Genet. 2015; 58(8): 409-415, doi: 10.1016/j.ejmg.2015.06.010, indexed in Pubmed: 26186913.

12. Davies MJ, Moore VM, Willson KJ, et al. Reproductive technologies and the risk of birth defects. N Engl J Med. 2012; 366(19): 1803-1813, doi: 10.1056/NEJMoa1008095, indexed in Pubmed: 22559061.

13. Sekulić SR, Ilić D, Novakov-Mikić A. Polyhydramnios and bone development: an unexplored relationship. Med Hypotheses. 2010; 75(3): 312314, doi: 10.1016/j.mehy.2010.03.011, indexed in Pubmed: 20347231.

14. Kornacki J, Adamczyk M, Wirstlein P, et al. Polyhydramnios - frequency of congenital anomalies in relation to the value of the amniotic fluid index. Ginekol Pol. 2017; 88(8): 442-445, doi: 10.5603/GP.a2017.0081, indexed in Pubmed: 28930371

15. Stanescu AD, Banica R, Olaru G, et al. Idiopathic polyhydramnios and fetal gender. Arch Gynecol Obstet. 2015; 291(5): 987-991, doi: 10.1007/s00404-014-3504-1, indexed in Pubmed: 25288271.

16. Kim TH, Kim JM, Lee HH. Questions about and speculations on the incidence of idiopathic polyhydramnios by fetal gender. Arch Gynecol
Obstet. 2015; 291(6): 1195, doi: 10.1007/s00404-015-3688-z, indexed in Pubmed: 25786637.

17. Richards RI, Sutherland GR. Dynamic mutations: a new class of mutations causing human disease. Cell. 1992; 70(5): 709-712, indexed in Pubmed: 1516128.

18. Güler B, Kılıç SH, Kızıltan MY. Variable genetic penetrance of myotonic dystrophy following the diagnosis of idiopathic polyhydramnios. Int J Gynaecol Obstet. 2016; 134(1): 103, doi: 10.1016/j.ijgo.2016.01.004, indexed in Pubmed: 27039051.

19. Rudnik-Schöneborn S, Zerres K. Outcome in pregnancies complicated by myotonic dystrophy: a study of 31 patients and review of the literature. Eur J Obstet Gynecol Reprod Biol. 2004; 114(1): 44-53, doi: 10.1016/j. ejogrb.2003.11.025, indexed in Pubmed: 15099870.

20. Yee C, Choi SJ, Oh SY, et al. Clinical characteristics of pregnancies complicated by congenital myotonic dystrophy. Obstet Gynecol Sci. 2017; 60(4): 323-328, doi: 10.5468/ogs.2017.60.4.323, indexed in Pubmed: 28791262.

21. Smits MJ, van Wijk MP, Langendam MW, et al. Association between gastroesophageal reflux and pathologic apneas in infants: a systematic review. Neurogastroenterol Motil. 2014; 26(11): 1527-1538, doi: 10.1111/nmo.12405, indexed in Pubmed: 25080836.

22. Pasquini L, Seravalli V, Sisti G, et al. Prevalence of a positive TORCH and parvovirus B19 screening in pregnancies complicated by polyhydramnios. Prenat Diagn. 2016; 36(3): 290-293, doi: 10.1002/pd.4769, indexed in Pubmed: 26970557.

23. Jakab A, Pogledic I, Schwartz E, et al. Fetal Cerebral Magnetic Resonance Imaging Beyond Morphology. Semin Ultrasound CT MR. 2015; 36(6): 465-475, doi: 10.1053/j.sult.2015.06.003, indexed in Pubmed: 26614130. 